chemotherapy significantly improved overall survival rates, without recurrence, and reduced the rate of distant metastatic dissemination. The objective of this work is to describe the histological response of cervical cancer treated with concomitant radiotherapy and chemotherapy (CCRT) followed by surgery, as well as the preoperative difficulties and morbidity related to surgery

Methodology This is a retrospective study of 126 patients treated for cervical cancer by CCRT followed by surgery at the Med VI Center for Gyneacologic and Breast Cancer Treatment at the UHC Ibn Rochd from January 2016 to December 2018.

Results The average age of the patients was 51, the mean total time from symptom onset to medical consultation was 7,5 months. Stage IIB was the discovery stage in $71 \%$ of the patients. Cervical biopsy results showed squamous cell carcinoma in 79\%, adenocarcinoma in $16 \%$ and $5 \%$ of patients had other histological types.

All of our patients received a weekly chemotherapy of $40 \mathrm{mg}$ of cisplatin, 4 cycles on average, associated with external radiotherapy sessions reaching 45 and 50 Gy supplemented by brachytherapy for 68 patients, $46 \%$ of patients were referred for surgery without additional brachytherapy most often due to lack of means. Surgical treatment, radical hysterectomy with salpingo-oophorectomy and bilateral pelvic lymphadenectomy was performed in $91.26 \%$ and $75.55 \%$ had a radical hysterectomy due to peroperative difficulties. The tumor residue was macroscopic in 29 patients. The surgical margins were positive in 8 cases. Parameters were invaded in 5 patients, 22 cases showed positive vascular emboles, Lymph node curage was positive in 14 cases.

Conclusion The overall treatment period is a main prognostic factor and second surgery following CCRT remains a great concern because of its morbidity.

Disclosures The authors declare they have no conflict of interest

\section{PREOPERATIVE BRACHYTHERAPY FOLLOWED BY LAPAROSCOPIC HYSTERECTOMY: A NEW OPTION TO CONSIDER FOR EARLY STAGES CERVICAL CANCER IN THE LIGHT OF THE LACC TRIAL RESULTS}

\footnotetext{
${ }^{1}$ Clemence Beyer, ${ }^{2}$ Houssein EL Hajj, ${ }^{3}$ Laurence Gonzague, ${ }^{3}$ Leonel Varela, ${ }^{3}$ Camille Jauffret, ${ }^{3}$ Guillaume Blache, ${ }^{3}$ Laura Sabiani, ${ }^{3}$ Gilles Houvenaeghel, ${ }^{3}$ Magalie Provansal, ${ }^{3}$ Renaud Sabatier, ${ }^{3}$ Eric Lambaudie. ${ }^{1}$ Grenoble Alpes University Hospital Center; ${ }^{2}$ Centre Léon Bérard; ${ }^{3}$ Paoli Calmettes Institute
}

\subsection{6/ijgc-2020-ESG0.44}

Objectives The aim of this study is to report the oncological and surgical outcomes of an alternative treatment strategy to upfront surgery for patients presenting with early stage cervical cancer (ESCC) (FIGO 2018 stages IA1-IB2). This treatment strategy consists of a combination preoperative brachytherapy (POBT) followed by a Querleu Morrow Type A laparoscopic or abdominal hysterectomy.

Material and Methods This retrospective study was conducted at the Marseille regional tertiary cancer center in France for patients treated for ESCC (FIGO 2018 stages IA1-IB2) between 2001 to 2012. All patients underwent a Low Dose Rate (LDR) POBT after confirming the absence of pelvic lymph node metastasis (radiological and surgical staging). 6 to 8 weeks after brachytherapy completion, all patients underwent a Querleu Morrow Type A hysterectomy (laparotomy and minimally invasive). The primary endpoint was the Disease Free Survival (DFS) and the secondary endpoint was the morbidity related to this radio-surgical multimodal approach.

Results A total of 138 patients were included. Histological analysis showed a complete response in 68 patients $(49.3 \%)$ and a residual tumor $<1 \mathrm{~cm}$ in 36 patients (26\%).

With a median follow up of 132 months (60 - 204 months), DFS was $93.5 \%$ and 9 recurrences occurred (1 local pelvic recurrence, 2 pelvic lymph node recurrences and 6 distant recurrences).

In univariate analysis, we found that a duration between the completion of brachytherapy and surgery exceeding 52 days is associated with a significant decrease in DFS ( $\mathrm{p}=$ 0.004$, OR $=8.5,95 \%$ CI $\{1.5 ; 48.7\})$. Pathological complete response was found to be associated with an increased DFS (p $=0.03 \mathrm{OR}=6.195 \% \mathrm{CI}\{1.8 ; 55.3\}$ ).

The brachytherapy related rate of late complications was $17.3 \% \quad(n=24) \quad$ (Chassagne glossary) and the surgery related urinary tract complications rate was $6.5 \%(n=9)$, with only 2 patients $(1.5 \%)$ presented grade 3 complications (Clavien Dindo classification).

Conclusion After a median follow up of 132 months, the multimodal radio-surgical management of ESCC (FIGO 2018 Stages IA1-IB2) consisting of POBT followed by a Querleu Morrow Type A laparoscopic hysterectomy appears to be a reasonable alternative to upfront open radical hysterectomy particularly in patients with high risk ESCC $(<2 \mathrm{~cm}$ associated with negative prognostic factors or for tumors measuring between 2 and $4 \mathrm{~cm}$ ).

This multimodal radio-surgical approach is associated with a low rate of complications and a reasonable rate of local recurrences compared to the results of the LACC trial. Further studies are necessary to confirm these results.

Disclosures Doctors Clémence Beyer, Houssein El Hajj, Laurence Gonzague, Leonel Varela Cagetti, Camille Jauffret-Fara, Guillaume Blache, Laura Sabiani, Magalie Provansal and Renaud Sabatier have no conflicts of interest or financial ties to disclose.

Gilles Houvenaeghel and Eric Lambaudie are proctors for Intuitive Surgical.

\section{ROBOTIC RADICAL PARAMETRECTOMY IN PATIENTS WITH UNDIAGNOSED INVASIVE CERVICAL CANCER: A STEP BY STEP PROCEDURE}

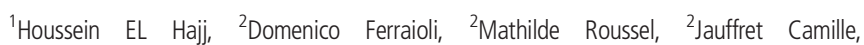
${ }^{2}$ Gilles Houvenaeghel, ${ }^{2}$ Eric Lambaudie. ${ }^{1}$ Centre Léon Bérard; ${ }^{2}$ Paoli Calmettes Institute

\subsection{6/ijgc-2020-ESGO.45}

Introduction/Background Occult discovery of invasive cervical cancer discovered after hysterectomy for non-malignant tumors is not uncommon. For patients presenting an incidental diagnosis of early stage invasive cervical cancer (FIGO Stages IA1IB2), two possible strategies can be proposed: Adjuvant radiation Therapy with no tumor target or Radical Parametrectomy (RP) associated with upper vaginectomy and pelvic lymph node dissection.

Methodology The procedure starts by incising the peritoneum on the lateral pelvic sidewall. Dissection is proceeded from the round ligament stump towards the ligated 